hafte Sache nach der Richtlinie über den Verbrauchsgüterkauf $^{23}$ keine Kosten entstehen dürfen. Insbesondere darf der Verkäufer keine Nutzungsentschädigung für das ausgetauschte Produkt verlangen.

Abschließend möchte ich noch kurz auf die Initiative der Kommission hinweisen, die vier wichtigsten Richtlinien des zivilrechtlichen Verbraucherschutzes zu einer gemeinsamen Regelung zusammenzufassen ${ }^{24}$. In Zukunft soll eine Richtlinie über die Rechte der Verbraucher die Haustürwiderrufsrichtlinie, die Richtlinie über missbräuchliche Klauseln, die Fernabsatzrichtlinie und die Verbrauchsgüterkaufrichtlinie ersetzen. Die neue Richtlinie soll abweichend vom früheren Konzept das nationale Recht vollständig harmonisieren. ${ }^{25}$ Die Systematisierung und Zusammenfassung der Regelungen ist sicher ein sinnvolles Vorhaben. Inwieweit die Kommission die Mitgliedstaaten von dem neuen Konzept der Vollharmonisierung überzeugen kann, bleibt allerdings abzuwarten.

\section{Schlussbemerkung und Ausblick - Was ändert Lissabon?}

Dieser allenfalls holzschnittartige Überblick macht deutlich, dass die gemeinschaftsrechtlichen Regelungen den Unionsbürgern die Verfolgung ihrer zivilrechtlichen Ansprüche in einer Gemeinschaft, in der 27 nationale Zivilrechtsordnungen koexistieren, erheblich erleichtert. Sie bestimmen, an welches Gericht sich der Bürger wenden kann, welches Recht in Fällen mit Auslandsbezug zur Anwendung kommt und setzen in vielen Vertragskonstellationen Mindeststandards zum Schutz der Verbraucher.

Sicher mag man die eine oder andere Regelung für zu dirigistisch und bevormundend oder aber für zu wirtschaftsliberal und nicht verbraucherfreundlich genug halten. Bei aller Kritik muss man sich aber vergegenwärtigen, dass ein Kompromiss gefunden werden muss, der in 27 Mitgliedstaaten akzeptiert wird. Zudem wird niemand bestreiten, dass die heutige Welt mit Internet und stark verflochtenem internationalen Handel weit komplizierter geworden ist als die Welt, die etwa die Verfasser des BGB vor Augen hatten.

Dennoch darf dies alles natürlich nicht zu einer überhasteten, inkohärenten und allein an der Tagesaktualität ausgerichteten Zivilrechtssetzung auf europäischer Ebene führen. Dies ist aber - glaube ich - bisher auch nicht der Fall. Insbesondere die Regelungen des internationalen Zivilprozessrechts (Brüssel I) und des internationalen Privatrechts (Rom I und II) sind gut aufeinander abgestimmt. Bedauerlich ist allenfalls, dass bisher ein kollisionsrechtliches Gegenstück zur Brüssel IIa VO fehlt.

Der Vertrag von Lissabon wird die Rechtsgrundlagen für die Rechtssetzung auf dem Gebiet des Zivilrechts kaum verändern. Dies ist aber auch nicht erforderlich, da die bisherigen Kompetenztitel völlig ausreichen. Eine wichtige Neuerung würde der Vertrag jedoch bringen: Die Beschränkung des Vorlagerechts auf letztinstanzliche Gerichte in Art. 68 EG würde entfallen. Dies wäre ein wichtiger Fortschritt für den Rechtsschutz des Einzelnen. Auch wenn sich die Notwendigkeit einer Vorabentscheidung zur Auslegung etwa der Rom I oder Rom II VO aufdrängt, müssen die Parteien derzeit einen langen Atem (und das nötige Geld) haben, um bis zur letzten Instanz durchzuhalten.

Denn eins ist klar: Ohne die einheitliche Auslegung der gemeinsamen Vorschriften durch die zentrale Instanz des Europäischen Gerichtshofs wäre die Schaffung eines Binnenmarkts mit einheitlichen rechtlichen Rahmenbedingungen nicht zu verwirklichen.

FOTOS: ALEXANDER LOUVET, 4/6 EVENT

23 Richtlinie 1999/44/EG des Europäischen Parlaments und des Rates vom 25. Mai 1999 zu bestimmten Aspekten des Verbrauchsgüterkaufs und der Garantien für Verbrauchsgüter, ABI. L 171, S. 12.

24 Vorschlag der Kommission vom 8. Oktober 2008 für eine Richtlinie des Europäischen Parlaments und des Rates über die Rechte der Verbraucher, KOM (2008) 614 endg.

25 Vorschlag der Kommission (zitiert in Fn. 24, S. 3).

\title{
Vereinbarkeit von Beruf, Familie und Privatleben
}

\section{Bericht über den Vorschlag der Europäischen Kommission zur Änderung des Mutterschaftsschutzes}

Die Gleichstellung von Frauen und Männern ist eines der grundlegenden Ziele der Europäischen Union, die durch deren Politiken erreicht werden soll ${ }^{1}$. Im Fahrplan der Kommission für die Gleichstellung von Frauen und Männern (2006-2010)² wird betont, dass Frauen und Männer im gleichen Maße am Arbeitsmarkt teilhaben sollen und die bessere Vereinbarkeit von Beruf, Privat- und Familienleben als eines Ziele mit hoher Priorität herausgestellt. Die Gleichstellung der Geschlechter ist auch ein Ziel der Europäischen Beschäftigungsstrategie (EBS) seit deren Einführung im November 1997. Maßnahmen zur Förderung der Vereinbarkeit von Berufs-, Privat- und Fa- milienleben, deren Bedeutung inzwischen allgemein anerkannt ist, sind heute aus wirtschaftlicher Sicht nach Ansicht der Europäischen Kommission unumgänglich geworden, da demografische Zwänge und Wettbewerbsdruck es immer dringlicher erscheinen lassen, die Arbeitsmarktbeteiligung der Frauen zu steigern. Außerdem korrelieren Geburtenrate und Arbeitsmarktbeteiligung positiv miteinander: In den Mitglied-

1 Artikel 2 und Artikel 3 Absatz 2 EG-Vertrag.

$2 \operatorname{KOM}(2006) 92$. 
staaten mit höherer Geburtenrate gibt es auch mehr erwerbstätige Frauen.

Mutterschaft beeinflusst die Beteiligung der Frauen am Arbeitsmarkt stark. Die Folge ist, dass die Beschäftigungsquote der Frauen mit unterhaltsberechtigten Kindern 2007 EU-weit nur 65,5 Prozent betrug, die der Männer dagegen 91,7 Prozent, ein Unterschied von mehr als 26 Prozentpunkten. 2007 sank die Beschäftigungsquote der Frauen zwischen 20 and 49 Jahren EU-weit um rund zwölf Prozentpunkte, sobald sie Kinder bekamen, während die der Männer um sieben Prozentpunkte anstieg.

Das Europäische Parlament hat wiederholt Verbesserungen der gemeinschaftlichen Rechtsvorschriften über Mutterschafts- und Elternurlaub angemahnt $^{3}$ : In seinen im Mai 2008 vorgelegten VorschlägenzurÄnderungder beschäftigungspolitischen Leitlinien fordert es die Bereitstellung von Erziehungsurlaubsmodellen und anderen Urlaubsmodellen.

Im Dezember 2007 konsultierte die Europäische Kommission die Mitgliedstaaten, um Informationen über deren Rechtsvorschriften zu allen Formen familienbezogener 'Urlaubs'regelungen/Familienzeiten zu erhalten und ihre Ansichten zu denkbaren Änderungen des gemeinschaftsrechtlichen Rechtsrahmens einzuholen. Ferner richtete die Kommission an mehrere europäische Nichtregierungsorganisationen einen Fragebogen, um deren Ansichten über die verschiedenen Optionen zur Änderung und Ergänzung der Rechtsvorschriften über Familienzeitregelungen im Zusammenhang mit der Vereinbarkeit von Berufs- und Familienleben in Erfahrung zu bringen. Außerdem wurden die Europäischen Sozialpartner in einem zweistufigen Verfahren zu möglichen Verbesserungen auf dem Gebiet der Work-Life-Balance befragt.

Derzeit existieren zwei Richtlinien, die den Urlaub aus familiären Gründen behandeln, nämlich die Richtlinie 92/85/EWG (die „Mutterschaftsrichtlinie“) und die Richtlinie 96/34/EG (die „Elternurlaubsrichtlinie“), die 1992 bzw. 1996 angenommen wurden. In ersterer ist der Anspruch auf mindestens 14 Wochen Mutterschaftsurlaub mit einem Entgelt mindestens in Höhe des Krankengeldes geregelt; in der letzteren ist ein dreimonatiger Elternurlaub für jedes Elternteil festgelegt, wobei der Elternurlaub in vielen Mitgliedstaaten von einem auf den anderen Elternteil übertragbar ist und hinsichtlich der Bezahlung keine Regelung getroffen ist.

Die Strategien der Mitgliedstaaten zur Förderung der Vereinbarkeit von Berufs- und Privatleben unterscheiden sich deutlich. So variiert die Dauer des Mutterschaftsurlaubs zwischen etwa 14 und 52 Wochen, wobei die entsprechenden Bezüge von 55 Prozent (mit Obergrenze) bis 100 Prozent (ohne Obergrenze) reichen. Der Anspruch auf Elternurlaub variiert zwischen drei Monaten und drei Jahren. Soweit bezahlter Elternurlaub vorgesehen ist, reicht dieser von 15 Tagen bis zu zwei Jahren.

Soweit es die Elternurlaubsrichtlinie angeht, verhandeln die europäischen Sozialpartner seit September 2008 über eine Neuregelung ihres Abkommens von 1995, das dieser Richtlinie zugrunde liegt. Es ist damit zu rechnen, dass ein neues $\mathrm{Ab}$ kommen im Juni unterzeichnet wird. Dieses wird voraussichtlich den Elternurlaub auf vier Monate pro Elternteil ausdehnen, wobei mindestens ein Monat nicht auf den anderen Elternteil übertragbar sein soll. Die Europäische Kommission wird diesem Übereinkommen sodann die Form einer Richtlinie geben.

Nach einer Folgenabschätzung ${ }^{4}$ wurde die überarbeitete Mutterschaftsrichtlinie am 2. Oktober 2008 von der Kommission im Rahmen eines sogenannten Vereinbarkeitspakets angenommen, das neben anderen Maßnahmen ${ }^{5}$ auch den Vorschlag zur Änderung der Mutterschaftsrichtlinie ${ }^{6}$ enthielt.

Die wesentlichen Änderungen im Vorschlag zur Änderung der Mutterschaftsrichtlinie sind die Folgenden:

- Verlängerung des Mutterschaftsurlaubs auf 18 Wochen

- Ein obligatorischer Zeitraum von sechs Wochen nach der Geburt des Kindes

- Mehr Flexibilität für die betroffene Frau, bis kurz vor der Geburt zu arbeiten

- Im Prinzip Bezahlung auf dem Niveau von 100 Prozent des vorherigen Gehalts

- Verstärkter Kündigungsschutz

- Ein Recht auf Anpassung der Arbeitszeiten und Arbeitsbedingungen

In der Erläuterung wird in dem Kommissionsdokument $^{7}$ dazu angegeben: Die Verlängerung „entspricht der Dauer, die in der im Jahr 2000 ange-

3 Zum Beispiel in der Entschließung 2003/2129(INI), P5_ TA(2004)0152), Nummer 26.

4 http://eur-lex.europa.eu/LexUriServ/LexUriServ.do?uri =SEC:2008:2596:FIN:EN:PDF.

5 Ein weiterer Richtlinienvorschlag betreffend die Richtlinie 86/613/EEC sowie einen Bericht über die Situation der Kindergartenplätze: http://ec.europa.eu/social/ main.jsp?langld=en\&catld $=89 \&$ newsld $=402 \&$ further News=yes.

6 http://ec.europa.eu/prelex/detail_dossier_real. cfm?CL=fr\&Dosld $=197460$.

7 http://eur-lex.europa.eu/LexUriServ/LexUriServ.do?uri =COM:2008:0637:FIN:DE:PDF.

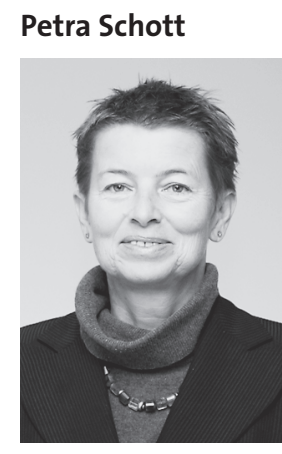

Ehemalige Richterin am Hess. VGH, seit 2004 Beamtin der Europäischen Kommission, derzeit zuständig für juristische Fragen im Bereich Chancengleichheit und Antidiskriminierung in der Generaldirektion „Beschäftigung, soziale Fragen und AntiDiskriminierung", Brüssel 
nommenen ILO-Empfehlung für den Mutterschutz vorgesehen ist, und soll ganz allgemein der Verbesserung von Gesundheit und Sicherheit von Frauen dienen, die ein Kind bekommen. Die Verlängerung des Mutterschaftsurlaubs soll dazu beitragen, dass Frauen sich besser von der Schwangerschaft und der Entbindung erholen, mehr Zeit mit ihren Kindern verbringen und länger stillen können.“

18 Wochen Mutterschaftszeit ist darüber hinaus bereits die Regel in zwölf Mitgliedstaaten, wie aus der Folgenabschätzung hervorgeht.

Eine sechswöchige obligatorische Zeit nach der Geburt entspricht der ILO Mutterschutz-Konvention C-183 aus dem Jahr 2000 (Artikel 4 Absatz 5). Mit der Regelung würde es den Mitgliedstaaten - im Unterschied zur bisherigen Regelung -unmöglich gemacht, obligatorische Zeiten vor der Geburt festzulegen, wie es bisher in vielen Fällen üblich ist. Damit ist die Frau flexibler, bis kurz vor der Geburt zu arbeiten und die gesamte Mutterschutzzeit nach der Geburt zu nehmen.

Hinsichtlich der Bezahlung wird nach dem zitierten Kommissionsdokument der Grundsatz festgelegt, „dass das volle vor dem Mutterschaftsurlaub bezogene monatliche Entgelt gezahlt wird. Dies ist allerdings nicht zwingend vorgeschrieben, da die Mitgliedstaaten gegebenenfalls für diese Zahlung eine Obergrenze einführen können, die aber nicht niedriger sein darf als das Krankengeld. Die Mitgliedstaaten können festlegen, ob die Bezüge während des Mutterschaftsurlaubs dem letzten monatlichen Arbeitsentgelt vor dem Mutterschaftsurlaub entsprechen oder einem über einen bestimmten Zeitraum berechneten Durchschnittsbetrag. “ 8

Weiterhin wird in dem bereits zitierten Kommissionsdokument zum Kündigungsschutz Folgendes ausgeführt: „Um der ständigen Rechtsprechung des Europäischen Gerichtshofs Rechnung zu tragen, wird vorgeschlagen, jegliche Vorbereitung einer möglichen Kündigung während des Mutterschaftsurlaubs außer in absoluten Ausnahmefällen zu verbieten. Nach der geltenden Richtlinie muss der Arbeitgeber nur dann schriftlich berechtigte Kündigungsgründe anführen, wenn einer Arbeitnehmerin während des Mutterschaftsurlaubs gekündigt wird. Mit der vorgeschlagenen Änderung wird diese
Pflicht des Arbeitgebers auf Fälle ausgedehnt, in denen einer Frau binnen sechs Monaten ab Ende des Mutterschaftsurlaubs gekündigt wird, sofern sie eine solche schriftliche Begründung verlangt. Mit dieser Bestimmung wird nicht die Absicht verfolgt, die Regeln für Einzel- oder Massenentlassungen zu ändern; es soll nur im Interesse sowohl des Unternehmens als auch der betroffenen Arbeitnehmerin festgelegt werden, dass während eines bestimmten Zeitraums nach der Rückkehr aus dem Mutterschaftsurlaub eine Kündigung schriftlich gebührend begründet werden sollte, wenn die Arbeitnehmerin dies wünscht.“

Ferner sollen Arbeitnehmerinnen „während des Mutterschaftsurlaubs oder bei der Rückkehr aus dem Mutterschaftsurlaub das Recht haben, ihren Arbeitgeber um eine Anpassung ihrer Arbeitszeiten und Arbeitsmuster an die neue familiäre Situation zu ersuchen, und dass Arbeitgeber verpflichtet sind, ein derartiges Ersuchen zu prüfen. Der Arbeitgeber ist aber nicht verpflichtet, dem Ersuchen zu entsprechen oder es weiterzuverfolgen. Die detaillierten Regeln für die Wahrnehmung dieses Rechts müssen von den Mitgliedstaaten festgelegt werden."

Der Richtlinienvorschlag wurde unter französischer und tschechischer Ratspräsidentschaft in den Ratsarbeitsgruppen diskutiert. Der Rat der Sozial- und Arbeitsminister hat den Vorschlag auf seiner Sitzung vom 9. März 2009 debattiert. Die tschechische Präsidentschaft wird auf der nächsten Sitzung der Sozial- und Arbeitsminister am 8. Juni 2009 über die erreichten Fortschritte berichten. (Anmerkung der Redaktion: Stand des Beitrags 19. Mai 2009)

Das Europäische Parlament hat bisher noch keinen Bericht mit einer Stellungnahme zu dem Richtlinienvorschlag der Kommission fertiggestellt.

Es wird erwartet, dass die am 1. Juli 2009 beginnende Ratspräsidentschaft Schwedens das Projekt vorantreiben und anstreben wird, zu einer gemeinsamen Lösung mit dem Europäischen Parlament zu kommen. ${ }^{8}$

8 http://eur-lex.europa.eu/LexUriServ/LexUriServ.do?uri=COM:2008: 0637:FIN:DE:PDF.

\section{Geparkt in der Kinder- und Jugendpsychiatrie}

Als Familienrichterin hatte ich in meinem Dezernat am Amtsgericht auch die Unterbringungssachen nach $\$ 1631$ b BGB zu bearbeiten. Es handelt sich dabei um die Unterbringung eines Kindes, die mit Freiheitsentziehung verbunden ist, also um die sogenannte „geschlossene Unterbringung“, die nur mit Genehmigung des Familiengerichts zulässig ist.

Der Antrag auf die Genehmigung wird meist von einem Elternteil gestellt, manchmal erreicht das Familiengericht auch nur ein Fax der Kinder- und Jugendpsychiatrie mit der Mittei- lung der Unterbringung eines Kindes. Es sind Eilfälle, die mich etwa fünf bis sechs Mal im Jahr erreichten. Da das Leben für Kinder, die in einer Kinder- und Jugendpsychiatrie (=KJPsychiatrie) aufgenommen werden müssen, in der Regel völlig aus dem Ruder gelaufen ist, sind meist auch die Eltern oder andere Personensorgeberechtigte so betroffen, dass sie nicht in der Lage sind, die Situation zu beherrschen. Sie sind nur froh, die Verantwortung für das Kind in der KJPsychiatrie lassen zu können. Aber: die geschlossene Unterbringung eines Kindes in 